\title{
Value of Wilms tumor gene product (WT-1) immunohistochemical expression in ovarian neoplasms
}

\author{
Amal Abd El hafez ${ }^{1 *}$, Ibrahim El-Dosoki ${ }^{1}$, Soheir Sirag ${ }^{1}$, Asmaa Gado ${ }^{1}$, Adel Denewer ${ }^{2}$ \\ ${ }^{1}$ Pathology Department, Faculty of Medicine, Mansoura University, Egypt \\ ${ }^{2}$ Surgical Oncology Department, Oncology Center, Mansoura, Egypt \\ *Corresponding author E-mail: amalabdelhafez@gmail.com
}

\begin{abstract}
Background: Ovarian cancer is a fatal malignancy among females worldwide. The wide morphologic variation among ovarian neoplasms poses diagnostic difficulties. Wilms' tumor gene protein (WT-1) is a recently added marker to the diagnostic panel of ovarian neoplasms and may have prognostic implications.

Objective: To evaluate the immunohistochemical expression of WT-1 protein in a broad array of ovarian neoplasms including metastases, identify its value in diagnosis and find out its association with the histopathological parameters including histological subtype, grade and FIGO stage.

Materials and methods: This retrospective study was conducted on 93 ovarian neoplasms collected from Pathology Department, Mansoura University and Mansoura Oncology Center. Immunohistochemistry was performed for WT-1 protein using the avidin-biotinperoxidase technique. Nuclear staining of tumor cells was considered as positive. Statistical analysis was performed using SPSS version 16.0.

Results: Significant statistical difference in WT-1 expression was observed among histological types of ovarian neoplasms ( $\mathrm{p}=0.04)$ being detected in $90 \%$ of serous tumors, in which significant statistical association was found between WT-1expression and grade $(\mathrm{p}=0.025)$, but not with stage. Most non-serous carcinomas were WT-1 negative. WT-1 reactivity was observed in tumors with Sertoli cell differentiation but undetectable in all other sex cord, germ cell, mixed germ cell-sex cord or metastatic tumors.

Conclusions: WT-1 is a highly specific marker for ovarian serous carcinomas versus other histological subtypes of ovarian neoplasms including metastases. It should be included in the immunohistochemical workup of carcinoma of unknown primary in women or in a panel to verify Sertoli cell differentiation in sex cord-stromal tumors.
\end{abstract}

Keywords: Immunohistochemistry, Ovarian Neoplasms, Serous Carcinoma, Sex-Cord, WT-1.

\section{Introduction}

Ovarian cancer is one of the leading problems in gynecologic malignancies in female's worldwide (Netinatsunthorn et al. 2006). Several histological types of epithelial ovarian cancer exist; approximately $60 \%$ are of the serous epithelial type (Kritpracha et al. 2005). Due to their nonspecific initial symptoms, $70 \%$ of patients have widespread metastatic disease at the time of diagnosis and consequently, the survival rate is generally low (Netinatsunthorn et al. 2006). Although with well-differentiated tumors the distinction between the morphological subtypes of ovarian neoplasms is straightforward, yet the distinction of poorly differentiated neoplasms may be difficult. This is of importance, since tumor typing is of prognostic significance and may be a predictor of likely chemoresponsiveness (Bagby et al. 2013, Fadare et al. 2013).

Wilms' tumor gene (WT-1), located on the short arm of chromosome 11 at $\mathrm{p} 13$, is a tumor suppresser gene and a nuclear transcription factor that is involved in the development of urogenital system (Cathro \& Stoler 2005, Zhao et al. 2007). WT-1 suppresses or activates a number of genes, including those for E-cadherin, PDGF- $\alpha$, EGF receptor, CSF-1, IGF-I, IGF-II receptor, c-myc, bcl-2, and WT-1 itself (Acs et al. 2004, Nakatsuka et al, 2006). The WT-1 protein also interacts with p53 to modulate the ability of E-cadherin and bcl-2 to Trans-activate their target genes (ACS
Et Al. 2004). Moreover, WT-1 protein is consistently detected in both normal ovarian germinal epithelium and human mesothelium (Chivukula et al. 2011).

Previous studies performed on this subject, revealed differences in WT-1 protein expression among various histological subtypes of ovarian neoplasms (Shimizu et al. 2000, Goldstein et al. 2001, AlHussaini et al. 2004, ACS et al. 2004, Waldstrøm \& Grove 2005, Fadare et al. 2013). In addition, WT-1 protein expression has been linked to unfavorable prognosis in patients with advanced serous epithelial ovarian carcinoma (Acs et al. 2004), thus the development of therapeutic agents to target WT-1 may provide an effective treatment option for ovarian cancer (Hsiao et al. 2010). Furthermore primary ovarian, peritoneal or tubal serous carcinomas were found to be usually WT-1 positive. In contrast, most uterine serous carcinomas are WT-1 negative (Goldstein \& Uzieblo 2002, Hashi et al. 2003, Al-Hussaini et al. 2004, Egan et al. 2004, Chivukula et al. 2011, Azueta et al. 2013, Bagby et al. 2013). Accordingly, when dealing with a disseminated serous carcinoma involving more than one site, reactivity with WT-1 favors a nonuterine primary (Hashi et al. 2003, Al-Hussaini et al. 2004, Egan et al. 2004, McCluggage 2006).

Nonetheless, during testing WT-1 expression, most studies have focused on surface epithelial ovarian tumors, though; very few studies have specifically investigated this marker in ovarian tu- 
mors of sex cord-stromal or germ cell derivation or in ovarian metastases. Yet, limited data in the literature suggested that WT-1 may be frequently expressed in sex cord-stromal tumors (Soleimanpour et al. 2011, Azueta et al. 2013).

The purpose of this work is to evaluate the immunohistochemical expression and the diagnostic value of WT-1 gene protein in a broad histological array of ovarian neoplasms including metastatic tumors, as well as studying the association between the histopathological parameters including; histological subtype, tumor grade and FIGO stage of positively immunostained neoplasms.

\section{Materials and methods}

\subsection{Patients and Clinicopathological evaluation}

This retrospective study was conducted on 93 ovarian neoplasms selected from the surgical pathology files of the Pathology Department in Mansoura University and Mansoura Oncology Center during the period from January 2006 to December 2007. The study included 41cases of surface epithelial-stromal tumors; 24 cases of sex cord-stromal tumors; 11 cases of germ cell tumors; 2 cases of mixed germ cell-sex cord tumors and 15 cases of metastatic tumors to the ovary. Inclusion criteria were the availability of tumor tissue paraffin blocks and the clinicopathologic data obtained from the medical records. None of the patients had received preoperative radiation or chemotherapy. Tumor- representative paraffin blocks were sectioned at $4-5 \mu \mathrm{m}$ and stained by haematoxyline and eosin (H\&E) stain to re-evaluate and subclassify tumors according to the latest World Health Organization classification, and grade them according to Gynecologic Oncology Group (GOG) system. Combination of clinical and histopathologic information was adopted for staging according to International Federation of Gynecology and Obstetrics (FIGO) staging criteria.

\subsection{Immunohistochemistry (IHC)}

After deparaffinization and rehydration, immunohistochemical staining for Wilms' tumor (WT-1) gene protein was performed on 4- to 5- $\mu \mathrm{m}$-thick tumor tissue sections on coated slides, using the standard avidin-biotin-peroxidase technique. Antibody used was WT-1 Ab-4 (Clone WLM04) mouse monoclonal antibody; Labvision corporation product, Fermont, CA. Cat. \#MS-1055-R7 $(7.0 \mathrm{ml})$ ready-to-use for immunohistochemical staining of formalin-fixed, paraffin-embedded tissues. Diaminobenzidin (DAB) was applied for visualization and hematoxylin for counterstaining. Positive control sections prepared from Wilms' tumor tissue as well as negative control sections of all tumors were processed with each IHC run.

\subsection{Evaluation of immunohistochemical staining}

Expression of WT-1 protein was visualized by observing the stained tissues under a light microscope and assessed by at least two pathologists.

\subsubsection{Staining positivity}

A semi-quantitative assessment of percentage of positive tumor cells was done. Staining was considered negative when no or less than $5 \%$ of tumor cells were stained, focal when $6-50 \%$ of tumor cells were positively stained and diffuse when more than $50 \%$ of tumor cells showed positive immunostaining.

\subsubsection{Staining intensity}

Intensity was scored as $1+$ (weak: staining is present but barely detectable), $2+$ (moderate), and 3+ (strong). Only moderate and strong immunostaining were considered positive.

\subsubsection{Cellular localization}

Positive WT-1 immunostaining was considered only when nuclear.

\subsection{Statistical analysis:}

Statistical analysis was carried out with the SPSS version 16.0 (Chicago, USA). Quantitative data were presented as number and percentage. Pearson Chi Square test $(\chi 2)$ was used for comparison between groups. Spearman (rank) correlation coefficient was used to calculate correlation between variables. $P$ value $\leq 0.05$ was considered statistically significant.

\section{Results}

\subsection{WT-1 expression in different histological types of ovarian tumors}

Table 1 shows the results of WT- 1 expression in 93 cases of ovarian neoplasms. Of the 41 cases of surface epithelial tumors, 22 cases $(53.7 \%)$ were positively stained. However, three cases of sex cord-stromal tumors (12.5\%) showed WT-1 immunoreactivity including a case of Sertoli cell tumor, a case of sex cord tumor with annular tubules (SCTAT) (Fig. 1) as well as a case of SertoliLeydig of intermediate differentiation (Fig. 2). WT-1 expression was undetectable in both germ cell tumors and mixed germ cell sex cord tumors. All metastatic tumors to the ovary of uterine, gastrointestinal tract, breast, hepatic and suprarenal origins were negative with WT-1. A significant statistical difference in WT-1 expression was observed among histological types of ovarian neoplasms $(p=0.04 ; \chi 2=8.19)$.

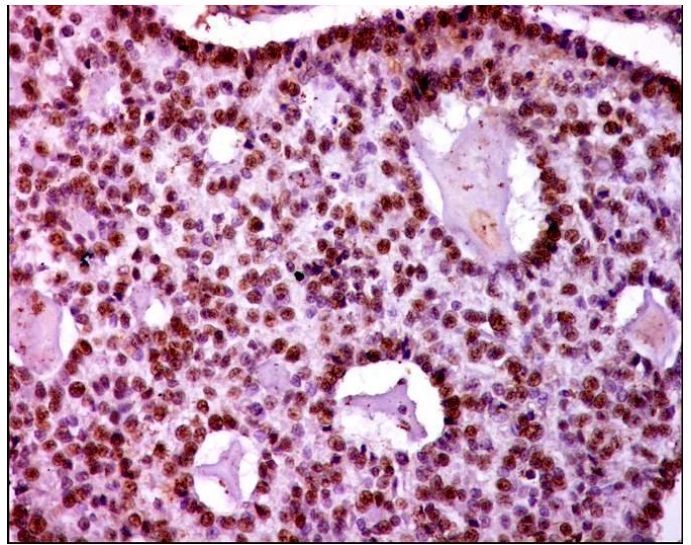

Fig. 1: Sex Cord Tumor with Annular Tubules (SCTAT) Demonstrating Strong Diffuse Nuclear Staining for WT-1 (IHC, X200).

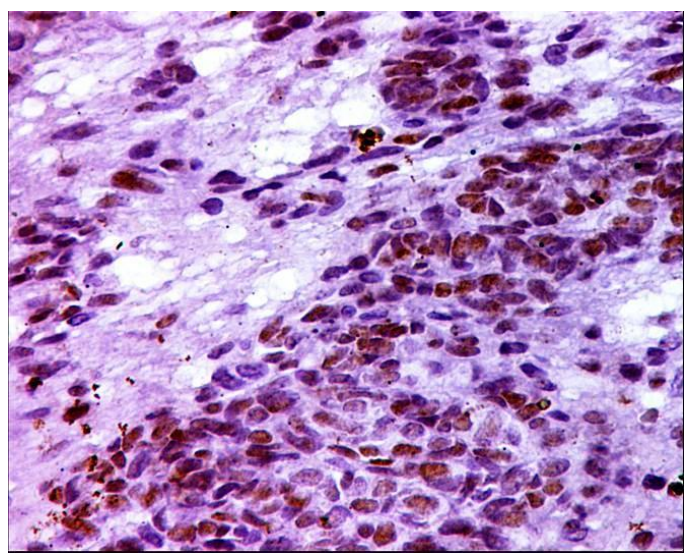

Fig. 2: Sertoli-Leydig Cell Tumor of Intermediate Differentiation Showing Moderate Focal Nuclear Staining For WT-1 in the Sertoli Cell Component (IHC, X400). 


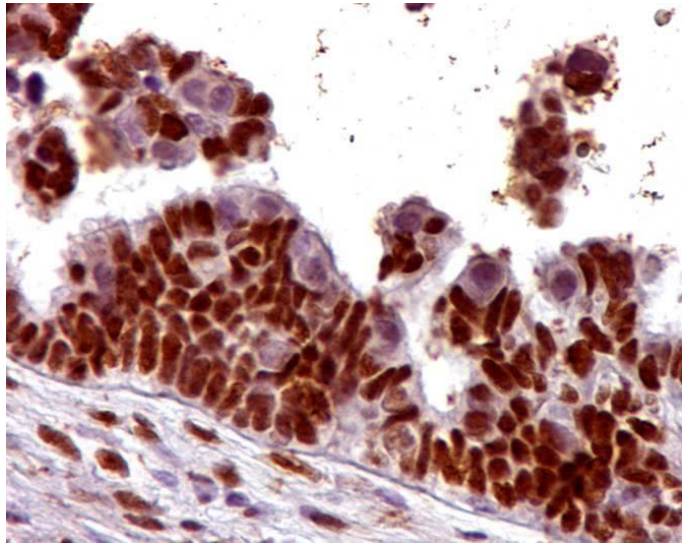

Fig. 3: Borderline Papillary Serous Tumor of the Ovary Showing Strong Diffuse Nuclear Staining for WT-1 (IHC, X400).

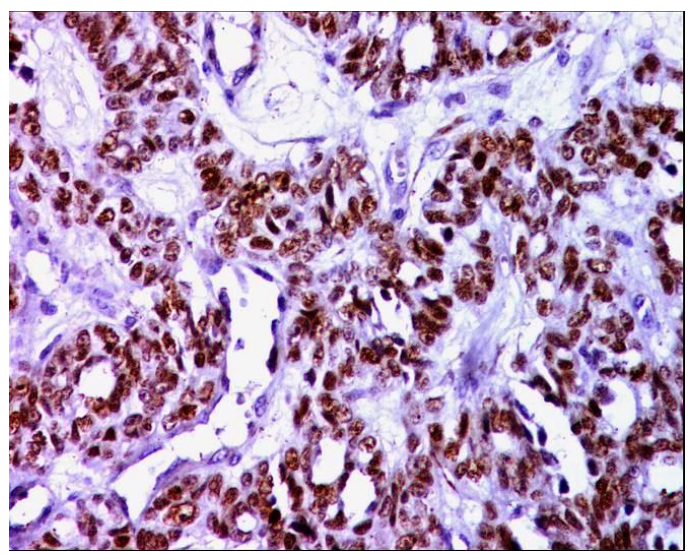

Fig. 4: Grade 1 Ovarian Papillary Serous Carcinoma Demonstrating Strong Diffuse Nuclear Staining for WT-1 (IHC, X200).

\subsection{WT-1 expression in different histological subtypes of ovarian surface epithelial tumors}

As shown in table 2, about $53 \%$ of surface epithelial tumors expressed WT-1. Among the 41 studied ovarian surface epithelial tumors, the only subtype that demonstrated remarkably positive expression of WT-1 was the ovarian serous tumors, in which 20 of 22 cases $(90.9 \%)$ were positive. Also one case of undifferentiated carcinoma and a case of endometrioid malignant mixed mullerian tumor (MMMT) showed focal WT-1 staining. Another case of undifferentiated carcinoma showed moderate cytoplasmic staining, which was not assessed as positive. Meanwhile, all other types of ovarian surface epithelial tumors were WT-1 non-reactive rendering a highly significant statistical difference regarding WT1 expression among surface epithelial tumors subgroups $(p<0.001$; $X_{2}=40.3$ ).

For ovarian serous carcinomas, most of cases $(72.7 \%)$ demonstrated diffuse strong nuclear positivity for WT-1. These findings contrast with non-serous ovarian carcinomas of which $89.5 \%$ of cases demonstrated no reaction for WT-1 (Table 3). Also, there was a highly significant statistical difference-regarding WT-1 expression-between serous and non-serous primary surface epithelial tumors $(p=0.001, \chi 2=23.3)$.

\subsection{Association between WT-1 expression and grade of malignant serous tumors}

Comparing positivity of WT-1 to histological grade of serous tumors (Table 4) revealed positive immunostaining of $80 \%$ of borderline serous tumors (Fig. 3), 83.3\% of grade I tumors (Fig. 4) and $100 \%$ of both grade II and III tumors (Fig. 5). There was a significant statistical association between WT-1expression and the grade of malignant serous surface epithelial tumors $(r=+0.54$ and $P=0.025)$ with tendency of WT- 1 expression to increase with increasing the grade of serous tumor.

Table 1: WT-1 Expression in Different Histological Types of Ovarian Neoplasms

\begin{tabular}{|c|c|c|c|c|c|}
\hline Histological type & Negative $(<5 \%)$ & Focal $(6-50 \%)$ & Diffuse $(>50 \%)$ & Total positive & $\mathrm{P}$ value \\
\hline Surface epithelial $(n=41)$ & $19(46.3 \%)$ & $6(14.6 \%)$ & $16(39.0 \%)$ & $22(53.7 \%)$ & \multirow{4}{*}{$0.04 *$} \\
\hline Sex cord-stromal $(n=24)$ & $21(87.5 \%)$ & $2(8.3 \%)^{\mathrm{a}}$ & $1(4.2 \%)^{b}$ & $3(12.5 \%)$ & \\
\hline Germ cell tumors and Mixed germ cell-sex cord tumors $(n=13)$ & $13(100 \%)$ & $0(0 \%)$ & $0(0 \%)$ & $0(0 \%)$ & \\
\hline Metastatic tumors $(n=15)$ & $15(100 \%)$ & $0(0 \%)$ & $0(0 \%)$ & $0(0 \%)$ & \\
\hline
\end{tabular}

value is significant if $<0.05$

${ }^{a}$ Sertoli cell tumor and Sertoli-Leydig cell tumor of intermediate differentiation.

${ }^{\mathrm{b}} \mathrm{Sex}$ cord tumor with annular tubules (SCTAT).

Table 2: WT-1 Expression in Different Histological Subtypes of Ovarian Surface Epithelial Tumors

\begin{tabular}{lllll}
\hline Histological subtype & Negative $(<5 \%)$ & Focal $(6-50 \%)$ & Diffuse $(>50 \%)$ & Total positive \\
\hline Serous $(\mathrm{n}=22)$ & $2(9.1 \%)$ & $4(18.2 \%)$ & $16(72.7 \%)$ & $20(90.9 \%)$ \\
Mucinous $(\mathrm{n}=10)$ & $10(100 \%)$ & $0(0 \%)$ & $0(0 \%)$ & $0(0 \%)$ \\
Endometrioid $(\mathrm{n}=5)$ & $4(80 \%)$ & $1(20 \%)$ & $0(0 \%)$ & $1(20 \%)$ \\
Clear cell $(\mathrm{n}=1)$ & $1(100 \%)$ & $0(0 \%)$ & $0(0 \%)$ & $0(0 \%)$ \\
Undifferentiated $(\mathrm{n}=2)$ & $1(50 \%)$ & $1(50 \%)$ & $0(0 \%)$ & $1(50 \%)$ \\
Transitional $(\mathrm{n}=1)$ & $1(100 \%)$ & $0(0 \%)$ & $0(0 \%)$ & $0(0 \%)$ \\
Total $(\mathrm{n}=41)$ & $19(46.3 \%)$ & $22(53.7 \%)$ & & \\
\hline
\end{tabular}

* $\mathrm{P}$ value is significant if $\leq 0.05$

Table 3: WT-1 Expression Difference between Serous and Non-Serous Ovarian Surface Epithelial Tumors

\begin{tabular}{lllll}
\hline Histological subtype & Negative $(<5 \%)$ & Focal $(6-50 \%)$ & Diffuse $(>50 \%)$ & Total positive \\
\hline Serous $(\mathrm{n}=22)$ & $2(9.1 \%)$ & $4(18.2 \%)$ & $16(72.7 \%)$ & $20(90.9 \%)$ \\
Non- serous $(\mathrm{n}=19)$ & $17(89.5 \%)$ & $2(10.5 \%)$ & $0(0 \%)$ & $2(10.5 \%)$ \\
\hline
\end{tabular}

* P value is significant if $\leq 0.05$

Table 4: Association between WT-1 Expression and Histological Grade of Primary Malignant Serous Tumors

\begin{tabular}{|c|c|c|c|c|c|}
\hline Histological grade & Negative $(<5 \%)$ & Focal $(6-50 \%)$ & Diffuse $(>50 \%)$ & Total positive & $\mathrm{P}$ value \\
\hline Borderline $(n=5)$ & $1(20 \%)$ & $1(20 \%)$ & $3(60 \%)$ & $4(80 \%)$ & $0.025^{*}$ \\
\hline Invasive carcinoma $(n=17)$ & $1(5.9 \%)$ & $3(17.6 \%)$ & $13(76.5 \%)$ & $16(94.1 \%)$ & \\
\hline Grade 1 serous carcinoma $(n=6)$ & $1(16.7 \%)$ & $2(33.3 \%)$ & $3(50 \%)$ & $5(83.3 \%)$ & \\
\hline Grade 2 Serous carcinoma $(n=3)$ & $0(0 \%)$ & $1(33.3 \%)$ & $2(66.7 \%)$ & $3(100 \%)$ & \\
\hline Grade 3 Serous carcinoma $(n=8)$ & $0(0 \%)$ & $0(0 \%)$ & $8(100 \%)$ & $8(100 \%)$ & \\
\hline
\end{tabular}

*P value is sign

$\mathrm{P}$ value is significant if $\leq 0.05$ 
Table 5: Association between WT-1 Expression and FIGO Stage of Primary Malignant Serous Tumors

\begin{tabular}{lllll} 
& Negative $(<5 \%)$ & Focal $(6-50 \%)$ & Diffuse $(>50 \%)$ & Total positive \\
\hline FIGO stage & $1(12.5 \%)$ & $2(25 \%)$ & $5(62.5 \%)$ & $7(87.5 \%)$ \\
Stage I $(\mathrm{n}=8)$ & $0(0 \%)$ & $1(25 \%)$ & $3(75 \%)$ & $4(100 \%)$ \\
StageII $(\mathrm{n}=4)$ & $1(12.5 \%)$ & $1(12.5 \%)$ & $5(62.5 \%)$ & $7(87.5 \%)$ \\
StageIII $(\mathrm{n}=8)$ & $0(0 \%)$ & $0(0 \%)$ & $2(100 \%)$ & $2(100 \%)$ \\
StageIV $(\mathrm{n}=2)$ & & & & 0.81 \\
\hline
\end{tabular}

\subsection{Association between WT-1 expression and FIGO stage of malignant serous tumors}

Comparing positivity of WT-1 to FIGO stage of serous tumors (Table 5) revealed staining of $87.5 \%$ of stage I tumors, $100 \%$ of stage II tumors and stage IV tumors and $87.5 \%$ of stage III tumors. No significant association was observed between WT-1 expression and FIGO stage of serous tumors $(r=-0.054$ and $P=0.81$ ).

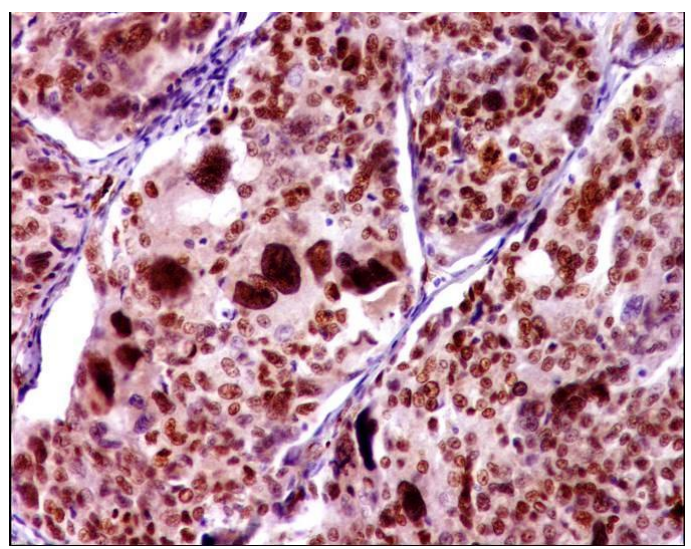

Fig. 5: Grade 3 Ovarian Papillary Serous Carcinoma Demonstrating Strong Diffuse Nuclear Staining for WT-1 (IHC, X200).

\section{Discussion}

Preferentially, WT-1 protein was found to be expressed in the genitourinary system and its aberrant expression has been implicated in the development of Wilms' tumor in this system. Recent studies have further shown that aberrant expression of the WT-1 protein may also be incriminated in the development and progression of other malignancies, including hematological malignancies, mesothelioma and breast myoepithelial and endothelial cell tumors (Nakatsuka et al. 2006, Hsiao et al. 2010). Our findings of WT-1 expression in ovarian neoplasms are consistent with a number of previous studies, which have shown that WT-1 is a useful marker for elucidation of ovarian serous tumors (Al-Hussaini et al. 2004, Köbel et al. 2009, Hsiao et al 2010, Chivukula et al. 2011, Bagby et al. 2013), being detected in more than $90 \%$ of malignant serous ovarian tumors in the present study. So, we suggest that WT-1 positivity within a poorly differentiated ovarian neoplasm, especially if widespread, may be a pointer towards serous carcinoma. This is of importance, since chemoresponsiveness may differ between different morphological subtypes of ovarian cancer (Hussaini et al. 2004, Chivukula et al. 2011).

Supported with other reports (Goldstein \& Uzieblo 2002, Acs et al. 2004, Hussaini et al. 2004, Cathro \& Stoler 2005, Netinatsunthorn et al. 2006, Nonaka et al. 2008, Bagby et al. 2013, Fadare et al. 2013), most serous carcinomas $(72.7 \%)$ demonstrated strong diffuse nuclear reaction, whereas, the non-serous neoplasms were almost always negative within the investigated cohort. However, the expression values of WT-1 ranged from $42 \%$ (Goldstein \& Uzieblo 2002), to 100\% (Waldstrøm \& Grove 2005) in different studies. The reasons for these discrepancies are unclear; nevertheless this may be explained by the use of different WT-1 antibodies (Cathro \& Stoler 2005), variation in the histolog- ical subtypes of tumors included or different cut-off values adopted for the staining positivity.

In agreement with our study, ovarian endometrioid carcinomas exhibited a very low rate of staining with WT-1 or were altogether negative in a number of studies (Shimizu et al. 2000, Al-Hussaini et al. 2004), although, Nonaka and his colleagues (2008) were able to detect WT-1 immunoreactivity in $27.8 \%$ of endometrioid carcinomas. The different WT-1 staining pattern between ovarian serous and endometrioid carcinoma stems from the fact that the underlying genetic events and the pathogenesis of these two neoplasms are different. Whilst serous carcinoma arises directly from the ovarian surface epithelium or from cortical inclusion cysts which are themselves WT-1 positive, in contrast, ovarian endometrioid carcinoma probably arises from ovarian endometriosis which is normally WT-1 negative (Feeley \& Wells 2001, AlHussaini et al. 2004).

Additionally, all mucinous and clear cell carcinomas included in this study were WT-1 negative. This finding correlates well with the results reported by other investigators (Goldstein \& Uzieblo 2002, Hashi et al. 2003, Waldstrøm \& Grove 2005, Hsiao et al. 2010, Azueta et al. 2013). In contrast, Shimizu et al. (2000), found some immunohistochemical expression of WT-1 in both mucinous and clear cell carcinomas, probably due to differences in the immunohistochemical protocols and the use of different primary antibody.

In the present study, one undifferentiated carcinoma exhibited WT-1 nuclear reactivity. In the same way, Waldstrøm and Grove (2005), demonstrated diffusely positive WT-1 expression in about half of undifferentiated carcinomas included in their study, reflecting that some of the histologically undifferentiated carcinomas are very low differentiated serous carcinomas, whereas the WT-1 negative carcinomas may be of other biological cell types. Similar to the later study (Waldstrøm \& Grove 2005), the single malignant transitional tumor in our group was WT-1 negative. Conversely, Logani et al., (2003) found a positive reaction for WT-1 in 14 of 17 transitional cell carcinomas of the ovary.

We reported a significant direct statistical association between WT-1expression and the grade of malignant serous surface epithelial tumors. This was in accordance with Al Hussaini et al. (2004), who demonstrated a lower frequency and intensity of tumor cell staining in borderline serous tumors versus carcinomas. While, Shimizu et al. (2000), were not able to establish such association. Genetic and pathogenesis differences may explain these findings. Most high grade serous carcinomas arise de novo from the ovarian surface epithelium or from inclusion cysts rather than through progression from a borderline tumor. In contrast, low grade serous carcinomas arise within either adenofibromas or borderline tumors through an intermediate stage of micropapillary carcinoma (Feeley \& Wells 2001, Singer et al. 2002, Singer et al. 2003, Al-Hussaini et al. 2004, Gilks 2004).

Few previous reports have demonstrated the ability of WT-1 gene expression to predict the stage in serous ovarian carcinoma (Netinatsunthorn et al. 2006). On the contrary our study showed no association between WT-1expression and FIGO stage of malignant serous surface epithelial tumors, which are similar to the findings of Shimizu and his colleagues (2000). Moreover, Netinatsunthorn et al. (2006) found an association between WT-1 gene expression and failure to respond to chemotherapy and overall survival but yet were unable to show any association with histological grade, or size of residual tumor. Our results may be attributed to the small number of stage II and stage IV patients investigated and so the data were incompatible with FIGO stage as a prognostic indicator. 
WT-1 gene product immunohistochemical expression has been described in ovarian sex cord-stromal tumors; however, results varied in the literature. In this study, three sex cord-stromal tumors showed WT-1 reactivity including a case of Sertoli cell tumor, a case of Sertoli-Leydig cell tumor (SLCT) of intermediate differentiation and a case of SCTAT, while all tested cases of granulosa cell tumor, thecoma and fibroma group were totally negative. Zhao et al.(2007) retrieved similar data with typically diffuse and strong immunostaining of Sertoli cells in most of cases. Conversely, one study demonstrated that WT-1 is frequently expressed in granulosa cell tumors but is negative in all cases of SLCT, fibroma, and thecoma (Cathro \& Stoler 2005). On the other hand, another study showed expression in all SLCT, SCTAT and most fibrothecomas and granulosa cell tumors (Deavers et al. 2003). In addition, Huiying et al.(2008), demonstrated that WT-1 is a fairly specific marker for ovarian fibromas, cellular fibromas and fibrothecomas. Accordingly, immunostaining for WT-1 is supposed to be useful in the distinction of pure Sertoli cell tumor from endometrioid carcinoma and carcinoid tumor (Huiying et al 2008), as well as helpful in the diagnosis of poorly differentiated SLCTs (Soleimanpour et al. 2011).

Because germ cell, mixed germ cell-sex cord tumors and metastatic tumors of different origins entirely lack WT-1 reactivity, as was seen in this study and comparable repots as well (Ordonez 2000, Goldstein et al. 2001, Hwang et al. 2004, Cathro \& Stoler 2005 , Han et al. 2010), it has been proposed that, WT-1 should be used in both histological and cytological material as one element of a panel of markers to distinguish ovarian carcinoma from germ cell tumors and carcinomas arising at other sites (Goldstein et al. 2001, Lee et al. 2002, Al-Hussaini et al. 2004, Nonaka et al. 2008, Azueta et al. 2013).

It has been formerly established that (Goldstein \& Uzieblo 2002, Al-Hussaini et al. 2004, Bagby et al. 2013), the immunoexpression pattern of WT-1 can significantly distinguish between endometrial carcinomas and ovarian serous carcinoma. In the present study, metastatic carcinomas of uterine origin were negative for WT-1, in contrast to all ovarian serous carcinomas. Therefore, immunohistochemical staining for WT-1 is recommended to diagnose ovarian and tubal high-grade serous carcinomas presenting in uterine samples (Fadare et al. 2013), and to determine the origin of peritoneally disseminated serous carcinomas.

In conclusion, WT-1 seems to be a sensitive and specific marker for diagnosis of ovarian serous tumors versus other histological subtypes of ovarian neoplasms including both gynecological and non-gynecological metastases. It should be included in the immunohistochemical workup of carcinoma of unknown primary in women especially when the tumor is poorly differentiated in patients with BRCA-1 mutations, WT-1 may be helpful in distinguishing serous carcinoma from breast carcinoma, a potential diagnostic dilemma. Virtually, most poorly differentiated ovarian carcinomas are serous in type; thus WT- 1 immunohistochemical staining may be useful in its verification. Moreover, WT-1 is detected in sex cord-stromal neoplasms with Sertoli cell differentiation, so it may be added as a part of a panel in diagnosing this group from its mimics, especially when poorly differentiated.

\section{Disclosure}

No relevant financial affiliations or conflicts of interest to disclose.

\section{References}

[1] Netinatsunthorn W, Hanprasertpong J, Dechsukhum C, Leetanaporn $\mathrm{R}$, Geater A (2006) WT1 gene expression as a prognostic marker in advanced serous epithelial ovarian carcinoma: an immunohistochemical study. BMC Cancer 11(6), 90-95.

[2] Kritpracha K, Hanprasertpong J, Chandeying V, Dechsukhum C, Geater A (2005) Survival analysis in advanced epithelial ovarian carcinoma in relation to proliferative index of MIB-1 immunostaining. J Obstet Gynaecol Res 31, 268-276.
[3] Bagby C, Ronnett BM, Yemelyanova A, Maleki Z, Kuhn E, Vang R (2013) Clinically occult tubal and ovarian high-grade serous carcinomas presenting in uterine samples: diagnostic pitfalls and clues to improve recognition of tumor origin. Int J Gynecol Pathol 32(5), 433443.

[4] Fadare O, James S, Desouki MM, Khabele D (2013) Coordinate patterns of estrogen receptor, progesterone receptor, and Wilms tumor 1 expression in the histopathologic distinction of ovarian from endometrial serous adenocarcinomas. Ann Diagn Pathol 17(5), 430-433.

[5] Cathro HP, Stoler MH (2005) the utility of calretinin, inhibin, and WT-1 immunohistochemical staining in the differential diagnosis of ovarian tumors. Hum Pathol 36(2), 195-201.

[6] Zhao C, Bratthauer GL, Barner R, Vang R (2007) Diagnostic utility of WT-1 immunostaining in ovarian sertoli cell tumor. Am J Surg Pathol 31(9), 1378-1386.

[7] Acs G, Pasha T, Zhang PJ (2004) WT-1 is differentially expressed in serous, endometrioid, clear cell, and mucinous carcinomas of the peritoneum, fallopian tube, ovary and endometrium. Int J Gynecol Pathol 23, 110-118.

[8] Nakatsuka S, Oji Y, Horiuchi T, Kanda T, Kitagawa M, Takeuchi T Kawano K, Kuwae Y, Yamauchi A, Okumura M, Kitamura Y, Oka Y, Kawase I, Sugiyama H, Aozasa K (2006) Immunohistochemical detection of WT-1 protein in a variety of cancer cells. Mod Pathol 19(6), 804-814.

[9] Chivukula M, Niemeier LA, Edwards R, Nikiforova M, Mantha G, McManus K, Carter G (2011) Carcinomas of distal fallopian tube and their association with tubal intraepithelial carcinoma: do they share a common "precursor" lesion? loss of heterozygosity and immunohistochemical analysis using PAX 2, WT-1, and P53 markers. ISRN Obstet Gynecol 2011, 858647. doi: 10.5402/2011/858647.

[10]Shimizu M, Toki T, Takagi Y, Konishi I, Fujii S (2000) Immunohistochemical detection of the Wilms tumor gene (WT-1) in epithelial ovarian tumors. Int J Gynecol Pathol 19, 158-163.

[11] Goldstein NS, Bassi D, Uzieblo A (2001) WT-1 is an integral component of an antibody panel to distinguish pancreaticobiliary and some ovarian epithelial neoplasms. Am J Clin Pathol 116(2), 246-252.

[12]Al-Hussaini M, Stockman A, Foster H, McCluggage WG (2004) WT1 assists in distinguishing ovarian from uterine serous carcinoma and in distinguishing between serous and endometrioid carcinoma. Histopathol 44, 109-115.

[13]Waldstrøm M, Grove A (2005) Immunohistochemical expression of Wilms' tumor gene protein in different histologic subtypes of ovarian carcinomas. Arch Pathol Lab Med 129(1), 85-88.

[14]Hsiao YH, Siddiqui S, Man YG (2010) Dual use of a single Wilms' tumor 1 immunohistochemistry in evaluation of ovarian tumors: a preliminary study of 20 cases. J Cancer 1, 93-97.

[15] Azueta A, Maiques O, Velasco A, Santacana M, Pallares J, Novell A, Llombart-Cussac A, Gonzalez-Tallada X, Mozos A, Prat J, Pillai R, Mata M, Matias-Guiu X (2013) Gene expression microarray-based assay to determine tumor site of origin in a series of metastatic tumors to the ovary and peritoneal carcinomatosis of suspected gynecologic origin. Hum Pathol 44(1), 20-28.

[16]Goldstein NS, Uzieblo (2002) WT-1 immunoreactivity in uterine papillary serous carcinomas is different from ovarian serous carcinomas. Is J Clin Pathol 117, 541-545?

[17]Hashi A, Yuminamochi T, Murata S, Iwamoto H, Honda T, Hoshi K (2003) Wilms' tumor gene immunoreactivity in primary serous carcinomas of the fallopian tube, ovary, endometrium and peritoneum. Int $\mathbf{J}$ Gynecol Pathol 22, 374-377.

[18]Egan JA, Ionescu MC, Eapen E, Jones JG, Marshall DS (2004) Differential expression of WT-1 and p53 in serous and endometrioid carcinomas of the endometrium. Int J Gynecol Pathol 23(2), 119-122.

[19] McCluggage WG (2006) Immunohistochemical and functional biomarkers of value in female genital tract lesions. Review. Int J Gynecol Pathol 25(2), 101-120.

[20]Soleimanpour H, Shirian S, Oryan A, Daneshbod K, Bagheri N, Daneshbod Y (2011) Cytologic, immunocytologic, histopathologic and immunohistologic diagnosis of the poorly differentiated sertolileydig cell tumor. Acta Cytol 55(4), 382-386.

[21]Köbel M, Kalloger SE, Carrick J, Huntsman D, Asad H, Oliva E, Ewanowich CA, Soslow RA, Gilks CB (2009) A limited panel of immunomarkers can reliably distinguish between clear cell and highgrade serous carcinoma of the ovary. Am J Surg Pathol 33(1), 14-21?

[22]Hwang H, Quenneville L, Yaziji L, Gown AM (2004) Wilms' tumor gene product: sensitive and contextually specific marker of serous carcinomas of ovarian surface epithelial origin. Appl Immunohistochem Mol Morphol 12 (2), 122-126.

[23]Nonaka D, Chiriboga L, Soslow RA (2008) Expression of pax8 as a useful marker in distinguishing ovarian carcinomas from mammary carcinomas. Am J Surg Pathol 32(10), 1566-1571. 
[24]Feeley KM, Wells M (2001) Precursor lesions of ovarian epithelial malignancy. Histopathol 38, 87-95.

[25]Logani S, Oliva E, Amin MB, Folpe AL, Cohen C, Young RH (2003) Immunoprofile of ovarian tumors with putative transitional cell (urothelial) differentiation using novel urothelial markers: histogenetic and diagnostic implications. Am J Surg Pathol 27(11), 1434-1441.

[26]Gilks CB (2004) Subclassification of ovarian surface epithelial tumors based on correlation of histological and molecular pathologic data. Int J Gynecol Pathol 23, 200-205.

[27]Singer G, Kurman RJ, Chang HW, Cho SK, Shih IeM (2002) Diverse tumorigenic pathways in ovarian serous carcinoma. Am J Pathol 160(4), 1223-1228.

[28][28] Singer G, Shih IeM, Truskinovsky A, Umudum H, Kurman RJ (2003) Mutational analysis of K-ras segregates ovarian serous carcinomas into two types: invasive MPSC (low-grade tumor) and conventional serous carcinoma (high-grade tumor). Int $\mathbf{J}$ Gynecol Pathol 22(1), 37-41

[29]Deavers MT, Malpica A, Liu J, Broaddus R, Silva EG (2003) ovarian sex cord-stromal tumors: an immunohistochemical study including a comparison of calretinin and inhibin. Mod Pathol 16(6), 584-590.

[30]Huiying H, Daniel JL, Pei H, Sean KL, Lawrence W, Peiguo GC (2008) Expression of CD56 and WT-1 in ovarian stroma and ovarian stromal tumors. Am J Surg Pathol 32(6), 884-890.

[31] Ordonez NG (2000) Value of thyroid transcription factor-1, Ecadherin, BG8, WT-1 and CD44S immunostaining in distinguishing epithelial pleural mesothelioma from pulmonary and nonpulmonary adenocarcinoma. Is J Surg Pathol 24, 598-606.

[32]Han L, Pansare V, Al-Abbadi M, Husain M, Feng J (2010) Combination of MUC5ac and WT-1 immunohistochemistry is useful in distinguishing pancreatic ductal carcinoma from ovarian serous carcinoma in effusion cytology. Diagn Cytopathol 38(5), 333-336.

[33]Lee BH, Hecht JL, Pinkus JL, Pinkus GS (2002) WT-1, estrogen receptor and progesterone receptor as marker for breast or ovarian primary sites in metastatic adenocarcinoma to body fluids. Is J Clin Pathol 117, 745-750. 